\title{
Hybrid density- and pressure-based splitting scheme for cavitating flows simulation
}

\author{
T. Alexandrikova, A. Pavlov \& V. Streltsov \\ Mentor Graphics Corporation, Mechanical Analysis Division, \\ Moscow, Russia
}

\begin{abstract}
A new numerical method to simulate steady-state isothermal liquid flows with hydraulic cavitation is proposed. The 3D averaged N-S equations with LamBremhorst $\kappa-\varepsilon$ turbulence model are used. The barotropic state equation is developed basing on thermodynamic equilibrium relations.

Simulation of such flows faces a lot of numerical difficulties concerned with variations of density, speed of sound and time scale.

The method is a hybrid splitting scheme that is a mixture of "density-based" and "pressure-based" approaches. The splitting scheme is the "pressure-based" SIMPLE-type algorithm in the region of incompressible liquid flow without cavitation. The scheme degenerates to the "density-based" algorithm in a compressible region (2-phase state or pure gas). The proposed method differs from both "density-based" preconditioned algorithms and SIMPLE-type methods adapted to the case of cavitating flows.

The method has been tested on numerous typical 3D problems with hydraulic cavitation. Results of numerical simulation are in good agreement with experimental data. The algorithm shows high efficiency for the considered problems. The method has been implemented in FloEFD ${ }^{\mathrm{TM}}$.

Keywords: cavitating flows, numerical method, splitting scheme.
\end{abstract}

\section{Introduction}

A new numerical algorithm for simulation of cavitating flows is proposed in the paper. Cavitating flows are encountered in a wide range of applications playing mostly negative but sometimes positive role. We consider hydraulic cavitation 
that occurs in liquid flows when the pressure falls close to the saturated vapour pressure. In this case a two-phase flow takes place.

An approach when fluid is considered as a homogeneous and isothermal medium is used. The medium has such thermodynamic properties that it can be either an incompressible liquid or a highly compressible medium that corresponds to twophase state or a compressible vapour that is treated as an ideal gas.

As cavitating flows are mostly turbulent, the averaged N-S equations are used in the paper. It is known that specific choice of turbulent model and its parameters influences on results of simulation (Senocak and Shyy [1]). However, this influence is not investigated in this paper because the problem requires a special research. To close the system of averaged N-S equations the LamBremhorst $k-\varepsilon$ turbulent model and barotropic state equation are utilized.

Constructing numerical method to simulate cavitating flows, it is important to take into account the fact that regions of incompressible flow and highly compressible flow exist simultaneously in calculation domain. The speed of sound may drop from thousands in liquid flow to units in vaporized flow. It leads to supersonic flows with high Mach numbers, sometimes with shocks. Thereby the cavitation problems are characterized by wide range of Mach number from near zero to several tens in one calculation domain.

An important feature of cavitating flows is the presence of several time scales. It is common that the time scale is greater by several orders of magnitude in liquid than in vaporized region with a supersonic flow. The typical time step varies over 2 orders of magnitude over calculation domain in considered below problems.

Another important feature is that properties of the model medium are changing dramatically when crossing the boundary an incompressible flow region and a compressible one. The speed of sound is changing by 3 orders of magnitude in considered problems. As a result, nonlinearity of the equation system strongly manifests itself on the boundary of liquid and vaporized regions.

Difficulties in numerical simulation of flows with widely varied Mach number appear not only in multiphase flows but in many traditional gas dynamics problems as well. Nowadays nature of the difficulties has been investigated in details. The problems are related to change of character of flow parameters mutual influence while Mach number is varying. In a compressible flow all parameters (density, pressure, momentum and temperature) significantly influence on each others. The form of the mass conservation equation is such that it is naturally interpreted as a density equation. So, the density, momentum and energy are used as basic unknowns when considering compressible flows. Such methods are named "density-based".

While Mach number approaching to zero, the pressure-density dependence becomes weak. So, in the case of low Mach number and incompressible flows, the mass conservation equation is used to describe the pressure-momentum coupling. Such methods are named "pressure-based".

To date there are two main approaches to calculate all-speed compressible flows. The first one employs the "density-based" methods originally developed to simulate speed compressible flows (usually at $\mathrm{M}>0.3$ ). The methods are 
adapted to low Mach number case by introducing artificial compressibility or using some preconditioning techniques (Kunz et al. [2], Li and Merkle [3], Li et al. $[4,5])$. Without such adaptations the methods are almost useless for lowspeed flows at $M \rightarrow 0$.

The second approach utilizes the "pressure-based" methods originally developed for incompressible flows. Usually these are SIMPLE-family splitting schemes (or "pressure-correction" methods) adapted to the case of compressible flows at high speed (Van der Heul et al. [6,7]).

Presented in the paper approach differs from the both abovementioned approaches. At first glance the idea to apply the "pressure-based" in region of incompressible flow and the "density-based" approach in region of supersonic compressible flow looks quite natural. But it is not obvious how to couple these approaches. We propose a way of combining the approaches that is based on the following simple key idea. Employing the finite volume method, we suggest mixing fluxes and pressure approximations that correspond to "pressure-based" and "density-based" approaches on faces of control volumes. After that these mixed approximations are substituted in SIMPLE-type splitting scheme. Managing the mixing weight in fluxes, we can obtain either the original SIMPLE-type semi-implicit splitting scheme or the explicit "density-based" scheme or a mixture of these approaches.

In this paper only the steady-state regimes of cavitating flows are considered. Such flows (for averaged flow parameters) are realized in a large number of applications. It is too consuming in time to solve such problems by an algorithm developed for time-dependent problems.

Of course, unsteady effects, such as periodically coming off cavitating vortex, are of considerable interest, but we will not consider such flows in the paper.

\section{Governing equations}

Mass conservation and momentum equations:

$$
\begin{gathered}
\frac{\partial \rho}{\partial t}+\frac{\partial\left(\rho u_{i}\right)}{\partial x_{i}}=0, \\
\frac{\partial \rho u_{i}}{\partial t}+\frac{\partial}{\partial x_{j}}\left(\rho u_{i} u_{j}\right)+\frac{\partial p}{\partial x_{i}}=\frac{\partial \tau_{i j}^{\Sigma}}{\partial x_{j}}+\rho \frac{\partial \varphi}{\partial x_{i}}-2 \cdot e_{i j k} \Omega_{j} \rho u_{k}, \\
\tau_{i j}^{\Sigma}=\left(\mu+\mu_{t}\right) s_{i j}, \tau_{i j}^{R}=\mu_{t} s_{i j}-\frac{2}{3} \rho k \delta_{i j}, \\
s_{i j}=\frac{\partial u_{i}}{\partial x_{j}}+\frac{\partial u_{j}}{\partial x_{i}}-\frac{2}{3} \delta_{i j} \frac{\partial u_{k}}{\partial x_{k}}, \varphi=\frac{1}{2} \Omega^{2} r^{2} .
\end{gathered}
$$

Here the Coriolis and centrifugal forces are taken into account.

Turbulence Lam-Bremhorst $k-\varepsilon$ model equations: 


$$
\begin{gathered}
\frac{\partial \rho k}{\partial t}+\frac{\partial \rho k u_{i}}{\partial x_{i}}=\frac{\partial}{\partial x_{i}}\left(\left(\mu+\frac{\mu_{t}}{\sigma_{k}}\right) \frac{\partial k}{\partial x_{i}}\right)+\tau_{i j}^{R} \frac{\partial u_{i}}{\partial x_{j}}-\rho \varepsilon \\
\frac{\partial \rho \varepsilon}{\partial t}+\frac{\partial \rho \varepsilon u_{i}}{\partial x_{i}}=\frac{\partial}{\partial x_{i}}\left(\left(\mu+\frac{\mu_{t}}{\sigma_{\varepsilon}}\right) \frac{\partial \varepsilon}{\partial x_{i}}\right)+f_{1} C_{\varepsilon 1} \frac{\varepsilon}{k} \tau_{i j}^{R} \frac{\partial u_{i}}{\partial x_{j}}-f_{2} C_{\varepsilon 2} \frac{\rho \varepsilon^{2}}{k} \\
\mu_{t}=C_{\mu} f_{\mu} \frac{\rho k^{2}}{\varepsilon}, f_{\mu}=\left(1-\exp \left(-0.0165 R_{y}\right)\right)^{2}\left(1+\frac{7.5}{\operatorname{Re}_{t}}\right), \operatorname{Re}_{t}=\frac{\rho k^{2}}{\mu \varepsilon} \\
f_{1}=1+\left(\frac{0.05}{f_{\mu}}\right)^{3}, f_{2}=1-\exp \left(-\operatorname{Re}_{t}^{2}\right), R_{y}=\frac{\rho \sqrt{k} y}{\mu}, \operatorname{Pr}_{t}=0.9 \\
C_{\mu}=0.09, C_{\varepsilon 1}=1.44, C_{\varepsilon 2}=1.92, \sigma_{k}=1, \sigma_{\varepsilon}=1.3 .
\end{gathered}
$$

It is assumed that flow is isothermal. So, the energy equation is omitted. It is supposed that $T$ is constant, $T=T_{0}$.

To close the system a state equation is needed. For practical purposes, it is important to be able to simulate flows of industrial liquids, such as liquid fuels, synthetic and mineral oils, freons and so on. But thermodynamic properties of such substances are incomplete or not widely available. Taking into account this fact, the state equation is derived in such a way that it requires just minimal and easy available parameters of a substance. We consider the barotropic state equation [27] for the homogeneous mixture consisting of liquid, its vapour and small amount of inert non-condensable gas (see Fig. 1). In practice, the most liquid fluids contain small amount of dissolved gases.

In Fig. 1 parameter $p_{E}^{L}$ is pressure value below which vapour appears in the mixture, $p_{E}^{V}$ is pressure value below which liquid disappears from the mixture. These two parameters $p_{E}^{L}$ and $p_{E}^{V}$ are derived from the condition of phases equilibrium employing Helmholtz thermodynamic potential [27]. In two-phase region the density-pressure dependency is

$$
\rho=\frac{p-p_{E}}{R T} \frac{M_{I}}{Y_{I}},
$$

where $p_{E}$ is the saturated vapour pressure, $M_{I}$ and $Y_{I}$ is the molecular mass and mass fraction of the inert gas.

Thus, defining only the density of a liquid and the saturated vapour pressure at temperature $T_{0}$, the molecular masses of substance of this liquid and a dissolved inert gas, we obtain the state equation for the specific fluid:

$$
\rho=\rho(p)
$$




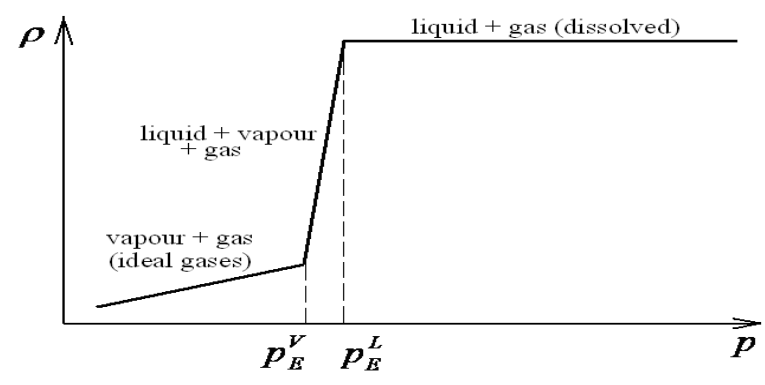

Figure 1: $\quad$ The barotropic state law $\rho(p)$.

\section{Numerical method}

Describing the numerical method, we pay the most attention to the operatorsplitting technique. Details of spatial approximations are omitted. Being introduced by Yanenko [28], the operator-splitting technique has been further developed to treat SIMPLE-type methods in terms of this approach. Now this technique is well-accepted in CFD. Details of specific treating are presented in Churbanov et al. [29].

Following the finite volume method, the integral form of conservation equations (1)-(4) is approximated.

Let us describe the principal idea how to couple two approaches, the $p$-based semi-implicit SIMPLE-type approach and the $\rho$-based explicit approach, in one numerical method.

Assume that there are two well-established numerical methods. One is $p$ based method for incompressible (low-compressible) flows, for example SIMPLE splitting scheme with corresponding spatial approximations of fluxes and pressure:

$$
\begin{aligned}
& F_{i n c}^{(\rho)}(m)=\sum a_{k} m_{k} \text { is face mass flux approximation, } \\
& F_{i n c}^{(m)}=u_{i+1 / 2} \cdot \sum b_{k} m_{k} \text { is face momentum flux approximation, } \\
& p_{i n c}=\sum c_{k} p_{k} \text { is face pressure approximation. }
\end{aligned}
$$

Here $m$ is momentum, $u$ is velocity, $a_{k}, b_{k}, c_{k}$ are approximation coefficients, and summation by index $k$ is performed over the stencils of the approximations. Subscript "inc" is used to refer to incompressible (low-compressible) case.

The second well-established method is $\rho$-based one for high-speed compressible flows. For example well-known AUSM (Liou) with corresponding spatial approximations of fluxes and pressure:

$$
\begin{aligned}
& F_{c o m p}^{(\rho)}=(\rho u)_{A U S M} \text { is the AUSM face mass flux approximation, } \\
& F_{\text {comp }}^{(m)}=(u \cdot m)_{A U S M} \text { is the AUSM face momentum flux approximation, } \\
& p_{\text {comp }}=(p)_{A U S M} \text { is the AUSM face pressure approximation. }
\end{aligned}
$$

Subscript "comp" is used to refer to compressible case. 
It is proposed that the coupling is to be performed by combining spatial approximations of convective fluxes and pressure, and substituting these mixed approximations into any SIMPLE-family scheme.

Spatial approximations of fluxes and pressure are mixed on CV (control volume) faces with some weight $w$. The weight is defined on CV faces and depends on state of fluid in adjacent CVs. It is equal to zero in liquid (incompressible flow region) and 1 in containing vapor medium (compressible flow region).

Note that the combined fluxes and pressure approximations are treated differently in time, as it is shown below. The $p$-based approximations are treated implicitly where it is necessary, and the $\rho$-based approximations are treated explicitly in all cases.

Unsteady form of equations is used to obtain steady-state solutions. But timestep is treated as calculation parameter that is varying in space. Symbol "^" is used to mark parameters that refer to new iteration and are treated as unknowns. Symbol " $\sim$ " is used to mark intermediate values of parameters. And parameters at old iteration do not have any "cup" symbol.

To simplify description, let us present deriving the method in $1 \mathrm{~d}$ case.

The discrete mass equation is written as

$$
\frac{V_{i}}{\Delta t_{i}} \delta \rho_{i}+\left(\hat{F}_{i+1 / 2}^{(\rho)}(\hat{m})-\hat{F}_{i-1 / 2}^{(\rho)}(\hat{m})\right) S=0,
$$

where $\delta \rho=\hat{\rho}-\rho$ and the weighted mass fluxes on $\mathrm{CV}$ faces are

$$
\begin{gathered}
\hat{F}_{i+1 / 2}^{(\rho)}(\hat{m})=\left(1-w_{i+1 / 2}\right) \cdot \hat{F}_{\text {inc }}^{(\rho)}(\hat{m})+w_{i+1 / 2} \cdot F_{\text {comp }}^{(\rho)}(m), \\
\hat{F}_{\text {inc }}^{(\rho)}=\sum a_{k} \hat{m}_{k}, \quad F_{\text {comp }}^{(\rho)}=(\rho u)_{A U S M} .
\end{gathered}
$$

Following the operator-splitting technique, the discrete momentum equation is written on two time levels, on intermediate and new ones. For "predictor", on intermediate time level, it is

$$
\frac{V_{i}}{\Delta t_{i}}\left(\widetilde{m}_{i}-m_{i}\right)+\left(\widetilde{F}_{i+1 / 2}^{(m)}-\widetilde{F}_{i-1 / 2}^{(m)}\right) S+\left(p_{i+1 / 2}-p_{i-1 / 2}\right) S=f_{i},
$$

where the weighted momentum fluxes and pressure on $\mathrm{CV}$ faces are

$$
\begin{gathered}
\widetilde{F}_{i+1 / 2}^{(m)}=\left(1-w_{i+1 / 2}\right) \cdot \widetilde{F}_{i n c}^{(m)}+w_{i+1 / 2} \cdot F_{c o m p}^{(m)}, \\
p_{i+1 / 2}=\left(1-w_{i+1 / 2}\right) \cdot p_{i n c}+w_{i+1 / 2} \cdot p_{c o m p}, \\
\widetilde{F}_{i n c}^{(m)}=u_{i+1 / 2} \sum b_{k} \widetilde{m}_{k}, \quad F_{\text {comp }}^{(m)}=(u \cdot m)_{A U S M}, \\
p_{i n c}=\sum c_{k} p_{k}, \quad p_{\text {comp }}=(p)_{A U S M} .
\end{gathered}
$$

Here $\widetilde{F}_{i+1 / 2}^{(m)}$ includes approximations of convective and diffusive fluxes of momentum. In $3 \mathrm{~d}$ case it also includes Coriolis force, and $f_{i}$ contains all other explicitly treated terms and forces.

The momentum equation is also written on new time level. The same $\widetilde{F}_{i \pm 1 / 2}^{(m)}$ but new pressure is employed: 


$$
\begin{gathered}
\frac{V_{i}}{\Delta t_{i}}\left(\hat{m}_{i}-m_{i}\right)+\left(\widetilde{F}_{i+1 / 2}^{(m)}-\widetilde{F}_{i-1 / 2}^{(m)}\right) S+\left(\hat{p}_{i+1 / 2}-\hat{p}_{i-1 / 2}\right) S=f_{i}, \\
\hat{p}_{i+1 / 2}=\left(1-w_{i+1 / 2}\right) \cdot \hat{p}_{i n c}+w_{i+1 / 2} \cdot p_{\text {comp }}, \\
\hat{p}_{i n c}=\sum c_{k} \hat{p}_{k}, \quad p_{\text {comp }}=(p)_{\text {AUSM }} .
\end{gathered}
$$

As usually for SIMPLE-type methods, an equation for pressure correction $\delta \hat{p}$ is derived to be used instead of the mass equation. For this purpose, eqn (7) is subtracted from eqn (8), and the result is substituted in the mass equation (6). This new equation replaces the mass equation in numerical method. Note that only equivalent algebraic transformations are employed, and the final system of discrete equations is fully equivalent to the original one.

Finally, the numerical method is written as the following equations that are solved consequentially.

The equation to calculate $\widetilde{m}$ (the "predictor" stage) is:

$$
\frac{V_{i}}{\Delta t_{i}}\left(\widetilde{m}_{i}-m_{i}\right)+\left(\widetilde{F}_{i+1 / 2}^{(m)}-\widetilde{F}_{i-1 / 2}^{(m)}\right) S+\left(p_{i+1 / 2}-p_{i-1 / 2}\right) S=f_{i},
$$

The equation to calculate the pressure correction $\delta p$ is:

$$
\frac{V_{i}}{\Delta t_{i}}\left(D_{p}^{(n)} \cdot \delta p^{(n+1)}\right)+\Delta\left(\delta p^{(n+1)}\right)=-\left(\widetilde{F}_{i+1 / 2}^{(\rho)}(\widetilde{m})-\widetilde{F}_{i-1 / 2}^{(\rho)}(\widetilde{m})\right) S,
$$

where $D_{p} \cdot \delta p$ is approximation of density correction $\delta \rho$, and

$$
\begin{gathered}
\Delta(\delta p)=\left(\left(1-w_{i+1 / 2}\right) F_{i n c, i+1 / 2}^{(\rho)}\left(\frac{\Delta t}{V} G_{i n c}(\delta p)\right)-\left(1-w_{i-1 / 2}\right) F_{i n c, i-1 / 2}^{(\rho)}\left(\frac{\Delta t}{V} G_{i n c}(\delta p)\right)\right) S, \\
G_{i n c}(\delta p)_{i}=\left(\left(1-w_{i+1 / 2}\right) \delta p_{i n c, i+1 / 2}-\left(1-w_{i-1 / 2}\right) \delta p_{i n c, i-1 / 2}\right) S \\
\delta p_{i n c, i+1 / 2}=\sum c_{k} \delta p_{k} .
\end{gathered}
$$

The equations to calculate new momentum (the "corrector" stage) and pressure are:

$$
\begin{gathered}
\hat{m}_{i}^{(n+1)}=\widetilde{m}_{i}-\frac{\Delta t_{i}}{V_{i}} G_{i n c}\left(\delta p^{(n+1)}\right)_{i}, \\
\hat{p}_{i}^{(n+1)}=p_{i}+\delta p_{i}^{(n+1)} .
\end{gathered}
$$

The state equation to calculate new density is:

$$
\rho^{(n+1)}=\rho\left(\hat{p}^{(n+1)}\right) \text {. }
$$

The coefficient $D_{p}$ is approximated using $\left(\frac{\partial \rho}{\partial p}\right)_{i}$ in $\mathrm{CV}$ and relations $\left|\frac{\rho_{i \pm 1}^{(n+1)}-\rho_{i}^{(n+1)}}{p_{i \pm 1}^{(n+1)}-p_{i}^{(n+1)}}\right|$ on CV faces.

The local time step is defined differently in incompressible and compressible flow regions: 


$$
\begin{gathered}
\Delta t_{i}=C_{i n c} \frac{h_{i}}{u_{i}} \text { in incompressible flow region, } \\
\Delta t_{i}=C_{\text {comp }} \frac{h_{i}}{u_{i}+c} \text { in compressible flow region, }
\end{gathered}
$$

here $c=\left(\frac{\partial \rho}{\partial p}\right)^{-1 / 2}$ is the sound speed, $C_{i n c} \approx 25, C_{\text {comp }} \approx 0.3$.

Such definition provide high rate of convergence to steady-state solution in both incompressible and compressible flow regions.

The internal iterations over index $n$ are introduced. These are needed to take into account non linear dependence of the density increment on the pressure increment is in vicinity of two values of pressure, $p_{E}^{L}$ and $p_{E}^{V}$. Three internal iterations were enough to provide robust calculations.

It is easy to see that setting $w=0$ leads to the standard SIMPLE (if only one internal iteration is performed).

Setting $w=1$ leads to the AUSM (with internal iterations).

The momentum equation:

$$
\begin{aligned}
& \frac{V_{i}}{\Delta t_{i}}\left(\tilde{m}_{i}-m_{i}\right)+\left((u \cdot m)_{A U S M, i+1 / 2}-(u \cdot m)_{A U S M, i-1 / 2}\right) S, \\
& \quad+\left(p_{A U S M, i+1 / 2}-p_{A U S M, i-1 / 2}\right) S=f_{i},
\end{aligned}
$$

The mass equation:

$$
\frac{V_{i}}{\Delta t_{i}}\left(D_{p}^{(n)} \cdot \delta p^{(n+1)}\right)+\left((\rho u)_{A U S M}-(\rho u)_{A U S M}\right) S=0,
$$

Other equations are as follows

$$
\hat{m}_{i}^{(n+1)}=\widetilde{m}_{i}, \quad \hat{p}_{i}^{(n+1)}=p_{i}+\delta p_{i}^{(n+1)}, \quad \rho^{(n+1)}=\rho\left(\hat{p}^{(n+1)}\right) .
$$

In the original AUSM method eqn. (18) is used for direct calculation of $\delta \rho$ and $\hat{\rho}=\rho+\delta \rho$. New pressure $\hat{p}$ is defined from the inverse form of the state equation $\hat{p}=p(\hat{\rho})$.

Iterating abovementioned algorithm by $\mathrm{n}$ we obtain the original AUSM method.

Thus, we have obtained the method that couples $p$-based and $\rho$-based approaches.

To perform described below calculations, the following specific spatial approximations were used. For "inc" approximations of convective fluxes in incompressible flow regions, a combination of the central and the $2^{\text {nd }}$ order upwind approximations was utilized. Limiters were used to provide monotonic properties of the approximation. For convective fluxes and pressure in compressible flow region well-known method AUSM [1] with some modifications as employed. For viscous fluxes central differences were used as usually. 
The proposed numerical method has shown high efficiency and robustness solving steady-state problems. This is due to the fact that different wellestablished approaches are used in incompressible and compressible regions of flow with corresponding different local time steps (15), (16).

\section{Results}

In this section we present some results of cavitating flows simulation.

\subsection{Centrifugal pump with two-dimensional curved blades}

The geometry of the pump is presented in Fig. 2. In details it is described in Coutier-Delgosha et al [9] as well as results of experimental and numerical investigation. For calculations we use numerical mesh with 72500 control volumes. Similarly to [9] we specify the following boundary conditions - fixed mass flow-rate at the inlet and fixed static pressure at the outlet boundaries respectively. Presented numerical method utilizes the local time step, which values are chosen taking into account the stability condition in the whole computational domain. The results of flow simulation in cavitating and noncavitating conditions were obtained.
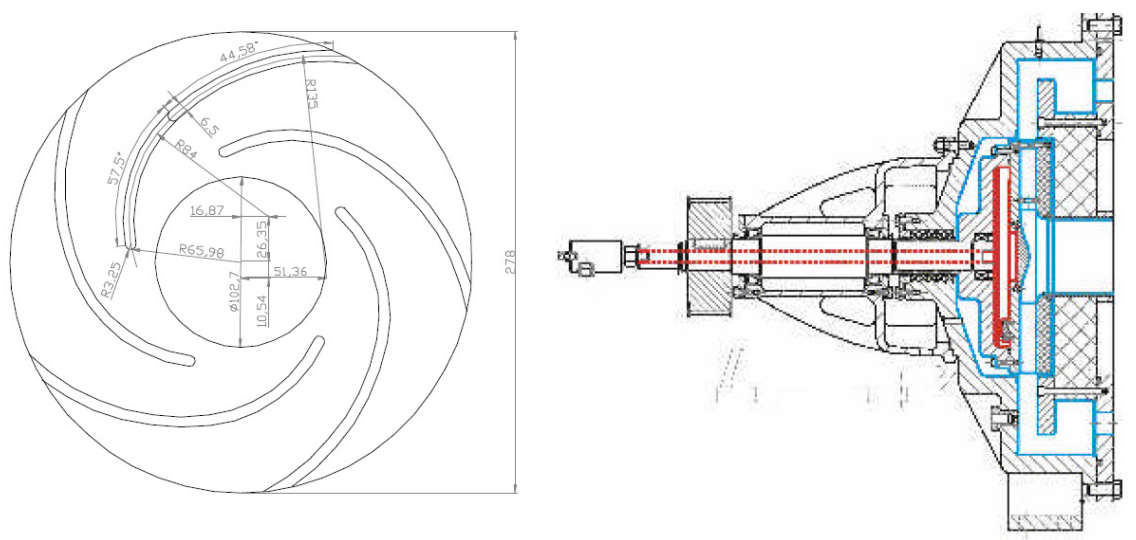

Figure 2: Centrifugal pump geometry, Coutier-Delgosha et al. [9].

The head drop chart H(NPSH) obtained by the calculation is drawn in Fig. 3. Calculations were performed considering various regimes in noncavitating and cavitating conditions, corresponding to various outlet pressure values and fixed mass flow-rate. The results were obtained for a large range of NPSH values, that correspond to different configurations of cavitation zones presented in the Fig. 4. The growth of cavitation zones and changes of their shape are observed with decrease of NPSH. Starting from NPSH=7 the cavitation zone attaching takes 


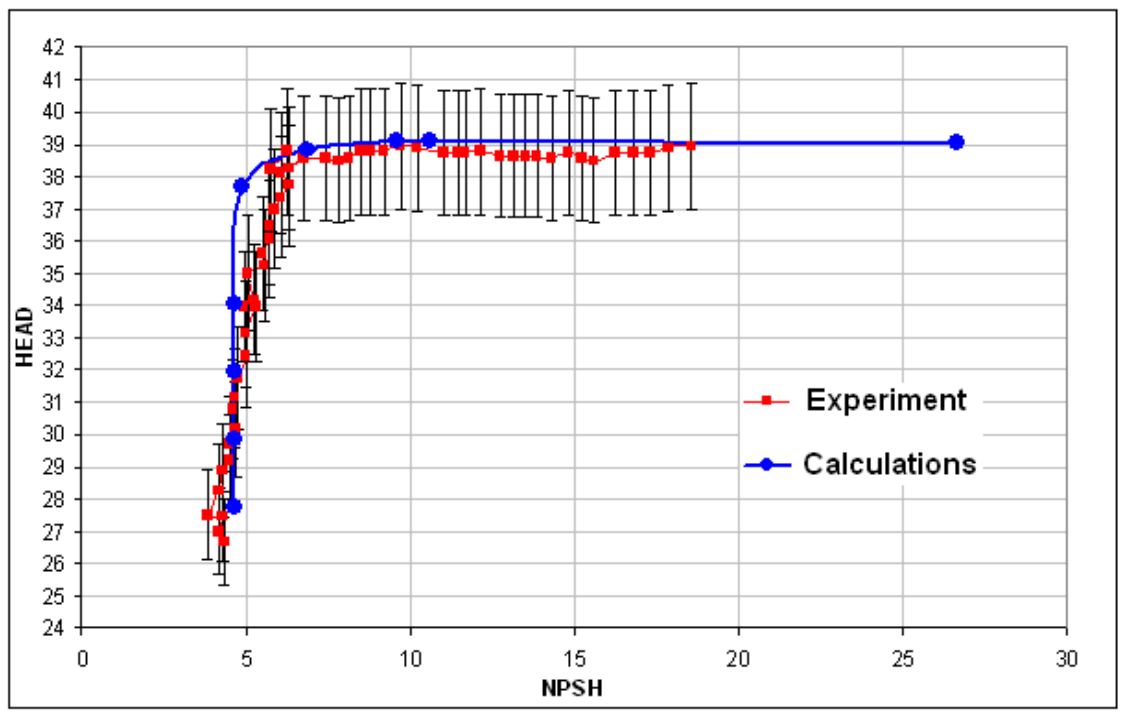

Figure 3: Head drop curves.

place on the outlet side of blades. Here H(NPSH) curve drop begins. With a further decreasing of NPSH the duct between blades is blocked by a cavity with a liquid-vapour mixture. Obtained numerical results are in a good agreement with experimental data.

\subsection{Liquid fuel flow in a throttle nozzle}

The results of liquid fuel flow in a throttle nozzle simulation are presented in this section. Model experiment for cavitating regimes of the flow investigation was described in detail in Winklhofer et al. [10]. In experiment the liquid fuel ran through a throttle made in the middle of a nozzle. A pressure drops were fixed and the authors measured mass flow-rates and visualized cavitation zones occurred in a narrow section. Detailed view of the throttle nozzle is shown in Fig. 5.

We simulated stationary 3D flows in a half of the geometry with a symmetry plane. As an inert attenuated gas air with mass fraction $\mathrm{Y}_{\mathrm{air}}=0.1 \%$ was chosen.

Numerical results were obtained for 9 various regimes with pressure drop $\mathrm{P}_{\text {in }}{ }^{-}$ $\mathrm{P}_{\text {out }}$ from $10^{6}$ to $9 \cdot 10^{6} \mathrm{~Pa}$, where $\mathrm{P}_{\text {in }}=10^{7} \mathrm{~Pa}$. The numerical and experimental data of flow rate are presented in Fig. 6. Marked on the figure range of ratio error is $5 \%$. 


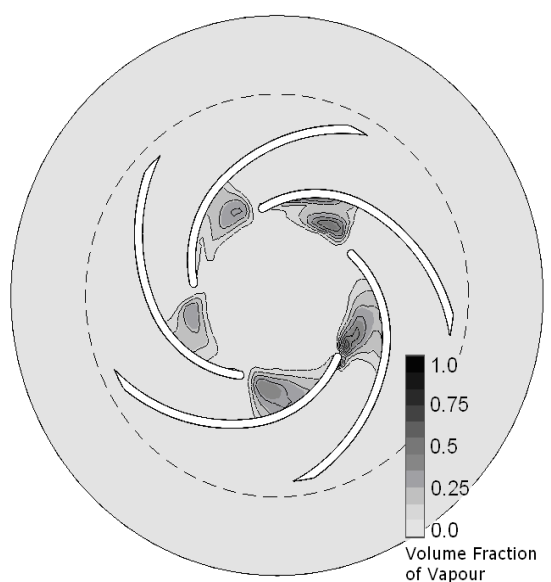

(a)

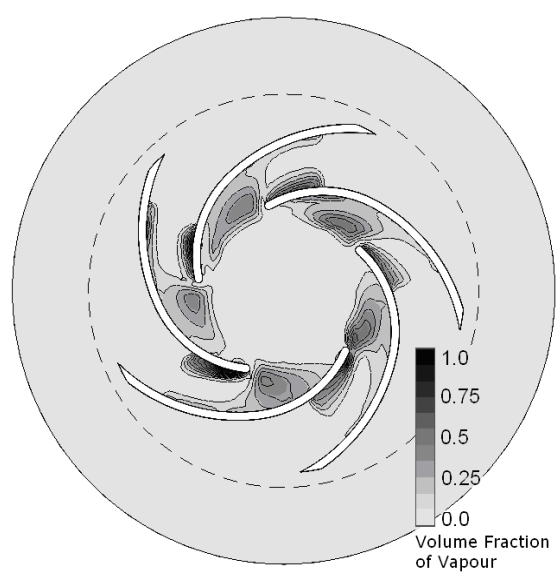

(c)

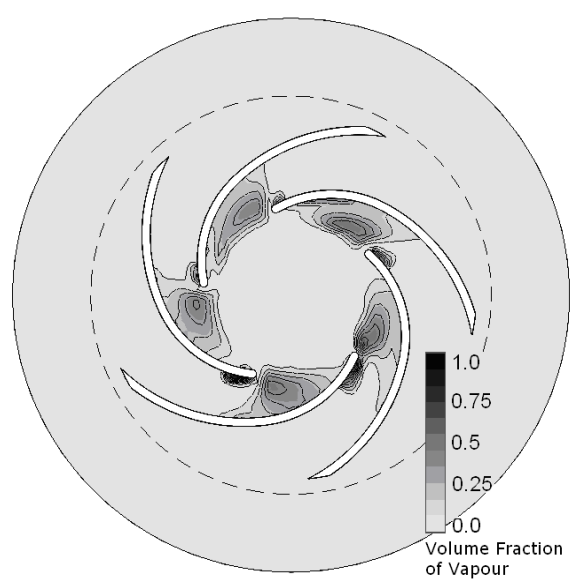

(b)

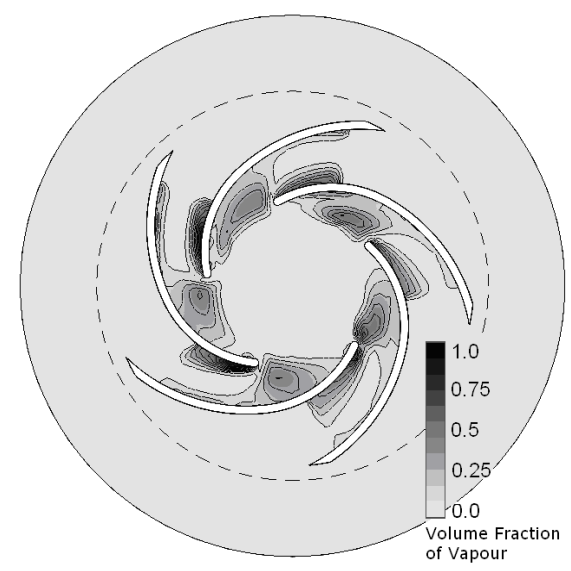

(d)

Figure 4: Volume fraction of vapour (a) $\mathrm{P}_{\text {out }}=4.2$ bar, (b) $\mathrm{P}_{\text {out }}=3.8$ bar, (c) $\mathrm{P}_{\text {out }}=3.4$ bar, (d) $\mathrm{P}_{\text {out }}=3.2$ bar.

\subsection{3-D multi-hole fuel injector}

We considered a model of car engine injector (Fig. 8) described in Schmidt et al. [11]. This model is axisymmetric so only $1 / 4$ of the model can be considered. To exclude influence of outlet boundary condition on a flow in the injector we added enlarged domain with boundary condition $\mathrm{P}=\mathrm{P}_{\text {out }}$ to the model outlet, just as in [11]. For this computational domain we used the mesh with $110000 \mathrm{CVs}$ 
52 Computational Methods in Multiphase Flow VI

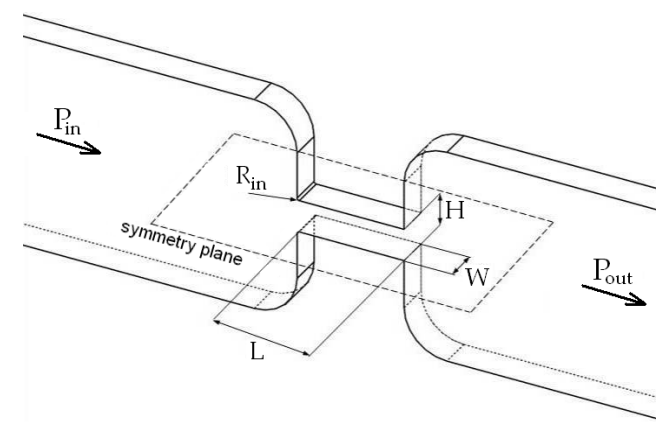

Figure 5: Throttle nozzle model. $\mathrm{L}=0.001 \mathrm{~m} ., \mathrm{H}=0.000299 \mathrm{~m} ., \mathrm{W}=0.0003 \mathrm{~m}$, $\mathrm{R}_{\text {in }}=0.00002 \mathrm{~m}$.

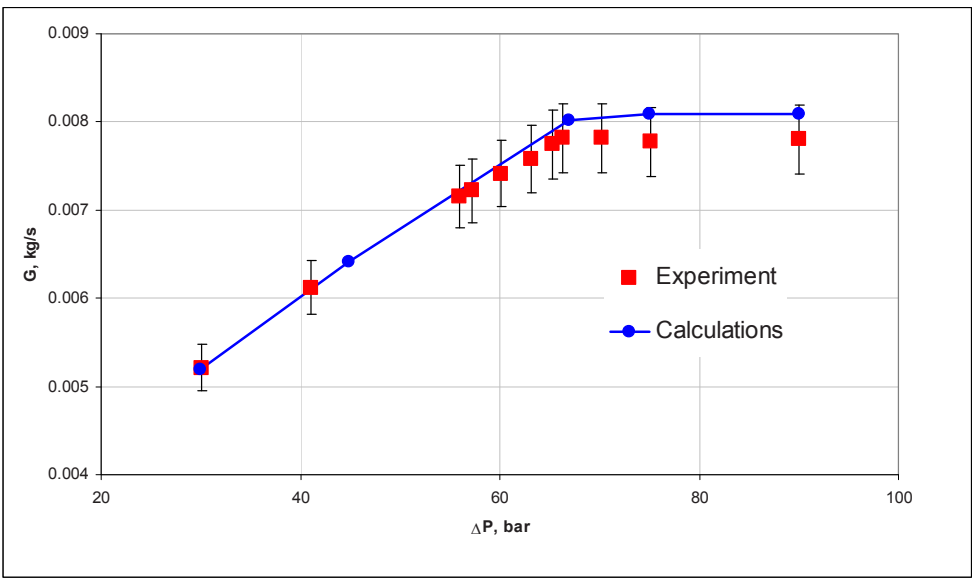

Figure 6: Flow rate characteristic of the throttle nozzle.

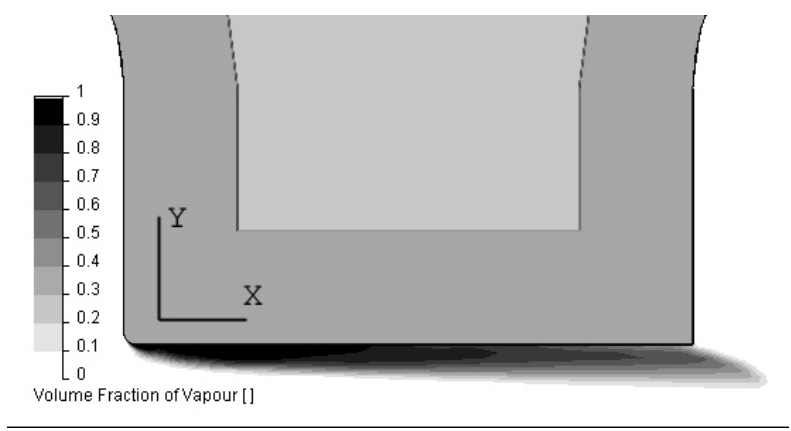

Figure 7: $\quad$ Volume fraction of vapour for pressure drop $\Delta P=9 \cdot 10^{6} \mathrm{~Pa}$ 


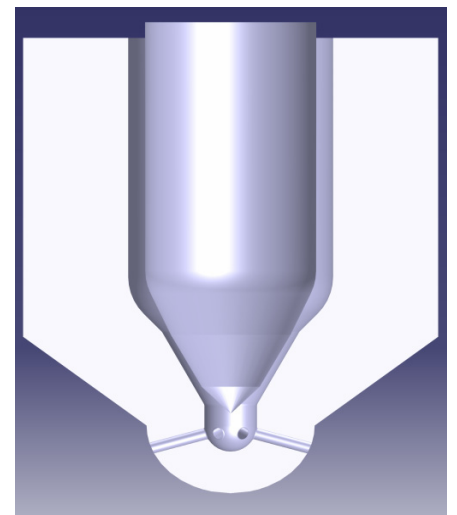

Figure 8: 180 degree section of the 3-D 6-hole injector geometry.

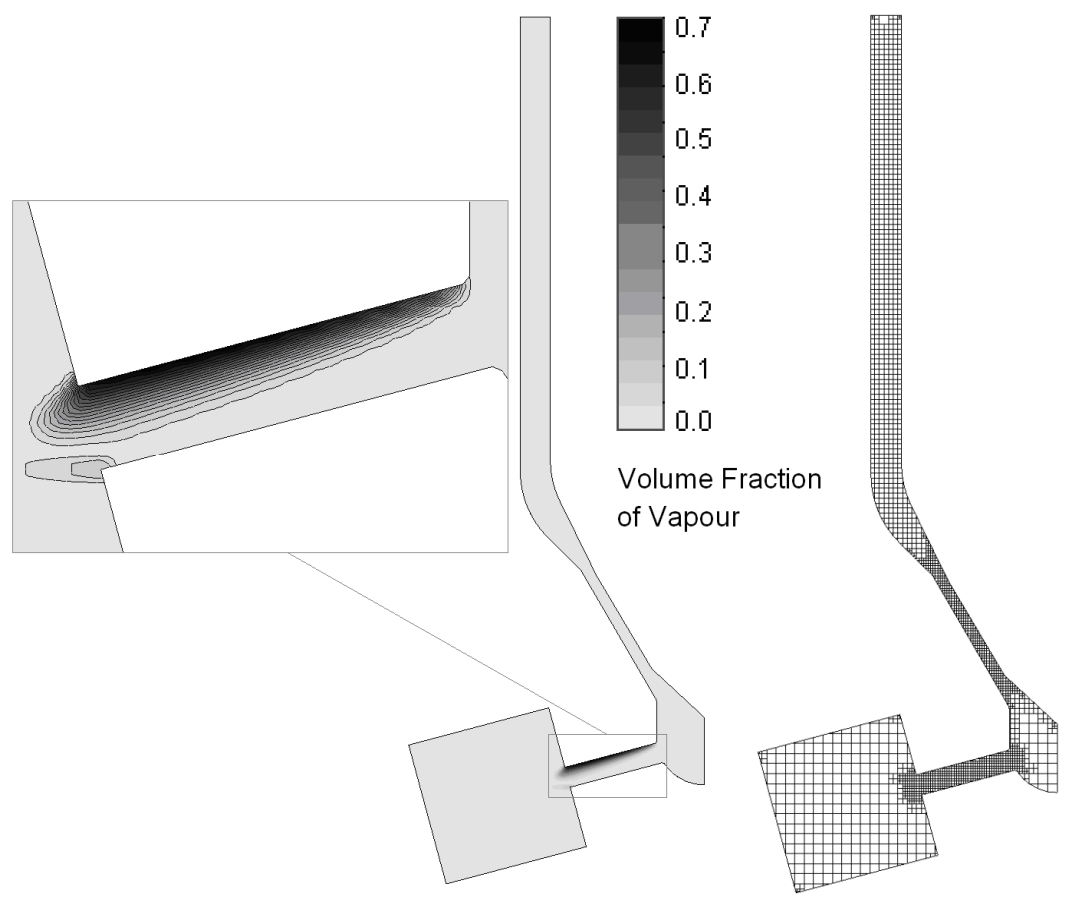

Figure 9: Mesh and steady-state cavitation zone. $p_{\text {in }}=600 \mathrm{~atm}, p_{\text {out }}=26 \mathrm{~atm}$, $T_{\text {init }}=333 \mathrm{~K}$.

(Fig. 9). Boundary conditions were defined by fixed values of pressure on the inlet and outlet boundaries, and different regimes were defined by different values of inlet pressure Pin, whereas outlet pressure was constant $P_{\text {out }}=26$ atm. Regime $\mathrm{P}_{\text {in }}=600$ atm, $\mathrm{P}_{\text {out }}=26 \mathrm{~atm}, \mathrm{~T}_{\text {init }}=333 \mathrm{~K}$ was considered. The volume 
fraction of vapour for stationary solution of this task is presented in Fig. 9. Comparison of numerical result with external data shows good accordance of mass flow $(60 \mathrm{~g} / \mathrm{s})$ for this regime. Cavitation zones also finely accord with calculations of Schmidt et al.

\section{Conclusions}

A hybrid method for simulating flows under hydraulic cavitation conditions is presented. It is based on coupling the well-established "pressure-based" and the "density-based" methods that allows efficient simulation of flows with both incompressible and highly compressible regions. Numerical experiments show that the new method is accurate, robust and efficient. It requires significantly less iterations compared to traditional "density-based" approaches to obtain a steadystate solutions.

Implemented in FloEFD ${ }^{\mathrm{TM}}$ method has been tested by solving a lot of typical 3D problems for cavitating flows with different liquids. Obtained results are in a good agreement with experimental data.

\section{Acknowledgments}

The authors thank Alexander V. Muslaev and Gennady E. Dumnov for helpful discussions and Alexander A. Sobachkin for continuous attention and support.

\section{References}

[1] Senocak I., Shyy W., A Pressure-Based Method for Turbulent Cavitating Flow Computations, Journal of Computational Physics 176, pp. 363-383 (2002)

[2] Robert F. Kunz, David A. Boger, David R. Stinebring, Thomas S. Chyczewski, Jules W. Lindau, Howard J. Gibeling, Sankaran Venkateswaran, T. R. Govindan, A preconditioned Navier-Stokes method for two-phase flows with application to cavitation prediction, Computers \& Fluids, Volume 29, Issue 8, 31 August 2000, pp. 849-875

[3] Li DING, Charles L. MERKLE, A unified framework for incompressible and compressible fluid flows, Journal of Hydrodynamics, Ser. B, Volume 18, Issue 3, Supplement 1, Proceedings of the Conference of Global Chinese Scholars on Hydrodynamics, July 2006, pp. 113-119

[4] Li Ding, Xia Guoping, Merkle Charles L., Large-Scale Multidisciplinary Computational Physics Simulations Using Parallel Multi-Zone Methods, In: Anil Deane, Akin Ecer, James McDonough, Nobuyuki Satofuka, Gunther Brenner, David R. Emerson, Jacques Periaux and Damien TromeurDervout, Editor(s), Parallel Computational Fluid Dynamics 2005, Elsevier, Amsterdam, 2006, pp. 227-234

[5] Li Ding, Xia Guoping, Merkle Charles L., Consistent properties reconstruction on adaptive Cartesian meshes for complex fluids 
computations, Journal of Computational Physics, Volume 225, Issue 1, 1 July 2007, pp. 1175-1197

[6] Van der Heul D.R., Vuik C., Wesseling P., A conservative pressurecorrection method for flow at all speeds. J.M. Burgers Center, Department of Applied Mathematical Analysis, Faculty of Information Technology and System, Delft University of Technology, Mekelweg 4, 2628 CD Delft, The Netherlands. 2002

[7] Van der Heul Duncan R., Vuik C. and Wesseling P., Efficient computation of flow with cavitation by compressible pressure, European Congress on Computational Methods in Applied Sciences and Engineering, ECCOMAS 2000

[8] Liou Meng-Sing, A Sequal to AUSM: AUSM+, Internal Fluid Mechanics Division, NASA Lewis Research Center, Cleveland, Ohio 44135, 1996

[9] Coutier-Delgosha O., Fortes-Patella R., Reboud J. L., Hofmann M., Stoffel B., Experimental and Numerical Studies in a Centrifugal Pump With TwoDimensional Curved Blades in Cavitating Condition. Transaction of the ASME, Vol. 125, November 2003, pp. 970-978

[10] Winklhofer E., Kull E., Kelz E., Morozov A. Comprehensive hydraulic and flow field documentation in model throttle experiments under cavitation conditions// ILASS-Europe annual meeting, 2001. pp. 574-579.

[11] Schmidt S.J., Sezal I.H., Schnerr G.H., Thalhamer M., Forster M. Compressible Simulation of Liquid/Vapour Two-Phase Flows with Local Phase Transition, 6th International Conference on Multiphase Flow, ICMF 2007, Leipzig, Germany, July 9-13, 2007

[12] Tryggvason Gretar, Bunner Bernard, Esmaeeli Asghar, Al-Rawahi Nabeel, Computations of Multiphase Flows, Advances in Applied Mechanics, Elsevier, 2003, Volume 39, pp. 81-120

[13] Coutier-Delgosha O., Fortes-Patella R., Reboud J.L., Hakimi N., Hirsch C., Stability of preconditioned Navier-Stokes equations associated with a cavitation model, Computers \& Fluids, Volume 34, Issue 3, March 2005, pp. 319-349

[14] Moukalled F., Darwish M., Sekar B., A pressure-based algorithm for multiphase flow at all speeds, Journal of Computational Physics, Volume 190, Issue 2, 20 September 2003, pp. 550-571

[15] Tseng Chien-Chou, Shyy Wei, Modeling for isothermal and cryogenic cavitation, International Journal of Heat and Mass Transfer, Volume 53, Issues 1-3, 15 January 2010, pp. 513-525

[16] Saurel Richard, Petitpas Fabien, Ray A. Berry, Simple and efficient relaxation methods for interfaces separating compressible fluids, cavitating flows and shocks in multiphase mixtures, Journal of Computational Physics, Volume 228, Issue 5, 20 March 2009, pp. 1678-1712

[17] Abgrall Remi, Saurel Richard, Discrete equations for physical and numerical compressible multiphase mixtures, Journal of Computational Physics, Volume 186, Issue 2, 10 April 2003, pp. 361-396 
[18] Metayer Le, Massoni J., Saurel R., Modelling evaporation fronts with reactive Riemann solvers, Journal of Computational Physics, Volume 205, Issue 2, 20 May 2005, pp. 567-610

[19] Saurel Richard, Abgrall Remi, A Multiphase Godunov Method for Compressible Multifluid and Multiphase Flows, Journal of Computational Physics, Volume 150, Issue 2, 10 April 1999, pp. 425-467

[20] Salvador F.J., Romero J.-V., Rosello M.-D., Martinez-Lopez J., Validation of a code for modeling cavitation phenomena in Diesel injector nozzles, Mathematical and Computer Modelling, Volume 52, Issues 7-8, Mathematical Models in Medicine, Business \& Engineering 2009, October 2010, pp. 1123-1132

[21] Jenny Patrick, Tchelepi Hamdi A., Lee Seong H., Unconditionally convergent nonlinear solver for hyperbolic conservation laws with Sshaped flux functions, Journal of Computational Physics, Volume 228, Issue 20, 1 November 2009, pp. 7497-7512

[22] Lowe C.A., Two-phase shock-tube problems and numerical methods of solution, Journal of Computational Physics, Volume 204, Issue 2, 10 April 2005, pp. 598-632

[23] Tang H. S., Sotiropoulos F., A Second-Order Godunov Method for Wave Problems in Coupled Solid-Water-Gas Systems, Journal of Computational Physics, Volume 151, Issue 2, 20 May 1999, pp. 790-815

[24] Bell John B, Colella Phillip, Trangenstein John A, Higher order Godunov methods for general systems of hyperbolic conservation laws, Journal of Computational Physics, Volume 82, Issue 2, June 1989, pp. 362-397

[25] Schwendeman D.W., Wahle C.W., Kapila A.K., The Riemann problem and a high-resolution Godunov method for a model of compressible two-phase flow, Journal of Computational Physics, Volume 212, Issue 2, 1 March 2006, pp. 490-526

[26] De Vault K.J., Gremaud P.A., Jenssen H.K., Numerical Investigation of Cavitation in Multidimensional Compressible Flows. SIAM Journal of Applied Mathematics, 2007, v:67, n:6, pp. 1675-1692

[27] Volkov V.A., Dergunov I.M., Muslaev A.V., Streltsov V.Y., Numerical simulation of cavitating flows in injectors using FloEFDTM. Papers of 5th Russian National Heat Transfer Conference., Moscow, 25-29 October 2010, Volume 1, pp. 73-76 (in Russian)

[28] Yanenko N.N., The Method of Fractional Steps: The Solution of Problems of Mathematical Physics in Several Variables, English translation edited by M. Holr. Springer-Verlag, New York, 1971.

[29] Churbanov A.G., Pavlov A.N. and Vabishchevich P.N., Operator-splitting methods for the incompressible Navier-Stokes equations on non-staggered grids. Part. 1: First-order schemes, Int. J. Numer. Metods Fluids, Vol. 21, 1995, pp. 617-40. 